\title{
Frequency Response Identification of the Dynamics of a Tokamak Plasma
}

A. Coutlis, D.J.N. Limebeer, J.P. Wainwright, J.B. Lister, P. Vyas

accepted for publication in IEEE Transactions on Control Science 


\title{
Frequency Response Identification of the Dynamics of a Tokamak Plasma
}

\author{
A. Coutlis, D.J.N. Limebeer and J.P. Wainwright, \\ Centre for Process Systems Eng. and Dept. of Electrical Eng., \\ Imperial College, London SW7 2BY, United Kingdom. \\ Email: a.coutlis@ic.ac.uk, d.limebeer@ic.ac.uk and j.wainwright@ic.ac.uk
}

\author{
J.B. Lister and P. Vyas \\ Centre de Recherches en Physique des Plasmas, École Polytechnique Fédérale de Lausanne, \\ Association EURATOM-Confédération Suisse, 1015 Lausanne, Switzerland. \\ Email: Jo.Lister@epfl.ch and Parag.Vyas@epfl.ch
}

\begin{abstract}
In this paper we describe the application of an $\mathcal{H}^{\infty}$ System Identification procedure to a Tokamak. The work is motivated by the need to create linear models which are suitable for controller design and which may be used to validate different models derived from physics principles. The paper develops an $\mathcal{H}^{\infty}$ System Identification algorithm and demonstrates its successful application to the Tokamak à Configuration Variable (TCV). Each of the required steps is detailed, from the design of identification experiments through to the creation of low-order models from a combination of Hankel model reduction and Chebycheff approximation. The method described in this paper is a worstcase identification technique, in that it aims to minimise the $\mathcal{H}^{\infty}$ error between the identified model and the true plant. Such a model is particularly well suited for robust controller design. The identified model of TCV is compared with various physics-based models.
\end{abstract}

\section{INTRODUCTION}

The limited supply of fossil fuels and the environmental risks associated with fission reactors are driving research into alternat,ve methods of electrical power production. One of the most interesting is the possibility of power generation using thermonuclear fusion. The easiest terrestrially achievable fusion reaction uses the Hydrogen isotopes Deuterium and Tritium; Deuterium occurs plentifully in sea water and Tritium may be extracted from Lithium. The reaction occurs at temperatures of the order of $10^{8} \mathrm{~K}$ and releases more energy per gramme than any other realistic fuel. The difficulties associated with handling the extremely hot fuel (which is in the plasma state) are compensated for by the facts that there are no greenhouse gases or the possibility of uncontrolled runaway reactions.

A Tokamak is a plasma confinement device which uses magnetic fields to restrict the motion of the plasma; a plasma is an ionised gas and therefore consists of charged particles. The basic principles of this device are illustrated in Figure 1. Essentially, a closed magnetic field is created from two separate magnetic field sources, a field which passes the long way around the torus (toroidal) and a field which passes the short way around the torus (poloidal). The toroidal field is generated by external field coils, while the poloidal field is generated by an inductively

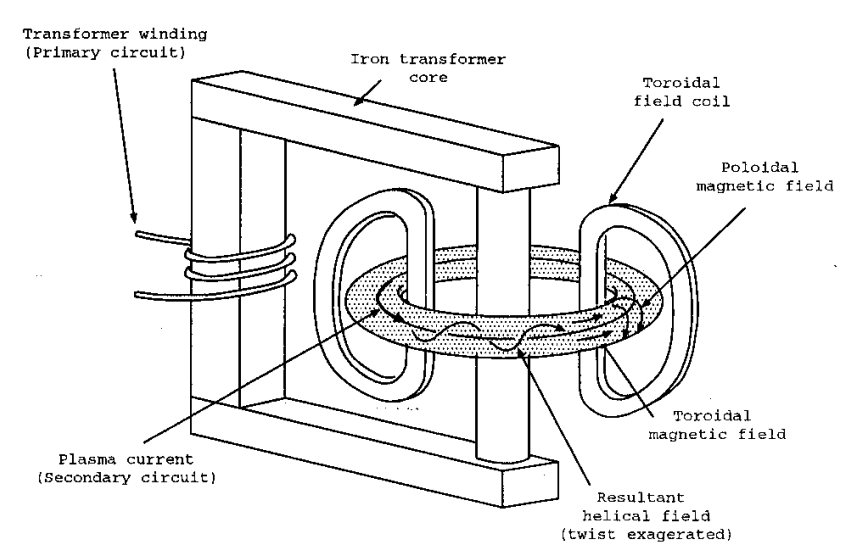

Fig. 1

A NAIVE TOKAMAK.

driven plasma current. Not shown in this figure are a set of toroidally wound coils which are used to position the plasma and stop it interacting with the containment vessel. These coils generate a poloidal magnetic field and are called the poloidal field coils (PF coils). They are situated outside of the plasma containment vessel and may be seen in Figure 2, which shows the poloidal cross-section of the Tokamak à Configuration Variable (TCV). The details of the construction of TCV can be found in Reference [1]

It is found that by increasing the plasma current both the plasma density and temperature are increased; this allows a machine to operate in more relevant fusion conditions. For a fixed toroidal field coil set, it is found that we can sustain a higher plasma current in a plasma which has an elongated cross-section. However, the process by which the plasma is elongated introduces an instability in the vertical plasma position.

A great deal of work has been performed on the dynamic modelling of the plasmas in tokamak fusion reactors [2],[3],[4],[5],[6]. The model validation has concentrated on either closed-loop comparisons [7] or open-loop 
experiments [8], but both of these approaches have disadvantages. Closed-loop comparison must obviously operate in the presence of a stabilising controller and the basic tokamak model is "screened" from observation, though the predictions and experimental results match excellently. The open-loop work necessarily leads to the destruction of the plasma since it is open-loop unstable with a fast growth rate, although once more the predictions and experimental results are in good agreement. The aim of this paper is to bridge the gap between the physics models referenced above and data measured from the TCV machine in a more satisfactory manner.

In this paper we describe how an $\mathcal{H}^{\infty}$ system identification technique was used to create a linear model of the response of a Tokamak plasma to voltages applied to the poloidal field coils. In this study we were particularly interested in the dynamics from the PF coils to a set of 6 parameters which describe the plasma position and shape. (The details may be found in Reference [6]). The plasmacoil system is multi-input multi-output (MIMO), continuous time and unstable, but for reasons detailed later, it was found more practical to treat it as a set of multi-input single-output (MISO) systems.

Tokamak inodels, be they identified or physics based, will play a key role in the design of control systems for future machines. For that reason, we based our studies on worst-case identification in $\mathcal{H}^{\infty}$, as models derived in this way require no a-priori order or model structure. Further, they have properties compatible with control system design studies. In regard, we refer the reader to the excellent survey articles [9],[10] and the references cited therein. We would also draw attention to the closed loop identification paper in that issue, which is of particular relevance [11]. The reader may wish to study an alternative two-stage frequency domain $\left(\mathcal{H}^{\infty}\right)$ identification procedure which has been used to identify models for lightly damped flexible structures [12].

In Section 2 we detail the identification algorithm for a MISO continuous-time system. The identification procedure uses data collected from a series of identification experiments. We design a multi-sinusoid signal which is applied to the inputs of the closed-loop system with signal frequencies selected using a bilinear formula. The method is based on the two-stage identification algorithms presented in [13],[14],[15],[16]. In the first stage, leastsquares approximation is used as a generalised DFT to obtain frequency response estimates from the time domain data. The second stage involves the use of a non-linear algorithm to identify a high-order non-causal FIR model. This is reduced to a low-order IIR identified model using Hankel model reduction and MISO Chebycheff approximation [17],[18],[19].

In Section 3 we outline a case study performed on TCV. We detail the experiments which, due to the plant instability, were performed in closed loop and show how the method outlined in Section 2 was used to create identified open-loop models of the machine. These identified models were compared with a range of physics models.
Finally, in Section 4 we present our conclusions.

\section{The Method}

\section{A. Experiment Design}

A suitable input signal is required for any identification experiment and in this subsection we detail the construction of our test signal, $s(t)$. It is chosen to be the sum of $N+1$ sine waves (including a DC term) and is of the form,

$$
s(t)=\sum_{i=0}^{N} M_{i} \cos \left(\omega_{i} t+\phi_{i}\right)
$$

in which

$$
\omega_{i}=\Omega_{o} \tan \left(\frac{\pi i}{2 L}\right), \quad i=0, \ldots, N, \quad N<L
$$

The amplitudes of the sine waves $\left(M_{i}\right)$ define how the energy of the test-signal is distributed between the measurement frequencies $\left(\omega_{i}\right)$, but they must be chosen so that the the test-signal keeps the plant within its linear range of operation. The phases $\left(\phi_{i}\right)$ are chosen to minimise the worst-case instantaneous amplitude of the test-signal for given $M_{i}$.

If it is impossible, or inappropriate, to use $\omega_{o}=0$, we replace $\omega_{0}$ with the smallest appropriate value. This smallest value will be used in the estimation of the plant's DC gain, so it should be chosen with reference to the expected system bandwidth.

The warping formula used to select the measurement frequencies is the same as that used in Reference [15]. The variables $\Omega_{o}$ and $L$ are chosen so that the frequencies they define span the "important region" of the system's frequency response. If useful, additional frequencies may be designed into the signal, for example around the closedloop bandwidth.

During an identification experiment, measurements are taken of the input and the output of the plant in its closed loop configuration, over a time interval which is longer than the period of the lowest-frequency sinusoid. If there are transient signals, these must be allowed to decay before the experimental data may be considered useful. A further constraint on the data is that the sampling frequency must be large enough to resolve the highest signal frequency.

If the system has $q$ inputs, it is necessary to perform $q$ separate experiments. These experiments must be independent, a notion to be defined in Section 2.3. One example of an independent set of experiments would be the case where the test-signal is applied to each input in turn.

\section{B. Curve Fitting}

If the system operates in a noise-free LTI manner, the frequencies of the spectral components in the measured signal will match exactly those in the test-signal. The frequency spectrum of the measured signals at these frequencies is obtained by least squares approximation. 
We choose the sines and cosines of the measurement frequencies $\left(\omega_{i}\right)$ as basis functions and define the approximation error,

$$
e_{k}=u\left(k t_{s}\right)_{i}-\sum_{i=0}^{N} B_{i} \cos \left(\omega_{i} k t_{s}\right)+C_{i} \sin \left(\omega_{i} k t_{s}\right),
$$

where $u\left(k t_{s}\right)$ is the measured signal, $t_{s}$ is the sampling time and $k=1, \ldots, m$ is a time index. We compute $B_{i}$ and $C_{i}$ $\left(C_{0}=0\right.$ if $\left.\omega_{0}=0\right)$ such that the square of the error $e^{T} e$ is minimised, where $e$ is the vector:

$$
e=\left[\begin{array}{llllll}
e_{1} & e_{2} & \cdots & e_{k} & \cdots & e_{m}
\end{array}\right]^{T} .
$$

The frequency component of the measured signal at $\omega_{i}$ is then defined by $A_{i} e^{j \phi_{i}}$, where

$$
A_{i}=\sqrt{B_{i}^{2}+C_{i}^{2}} \text { and } \phi_{i}=\tan ^{-1}\left(-C_{i} / B_{i}\right) \text {. }
$$

The amplitudes of the residuals $\left(e_{i}\right)$ which cannot be decomposed into the measurement frequencies are a measure of data corruption due to external disturbances, measurement noise and non-linearities of the system. Therefore, the smaller the residuals compared to the measured signals the greater the confidence in the results of the identification procedure.

\section{Frequency Response Estimation}

Using the technique detailed in the previous subsection, we estimate the frequency spectra of all of the input and output data collected during the identification experiments. To identify a $1 \times q$ MISO system we need to have performed $q$ :xperiments. For the $j$-th experiment we define the input frequency spectrum as $U_{k}^{j}\left(\omega_{i}\right)$ and the output frequency spectrum as $Y^{j}\left(\omega_{i}\right)$, where $k$ indexes the inputs. An estimate of the plant's frequency response at each measurement frequency is given by,

$$
\hat{G}\left(\omega_{i}\right)=\left[\begin{array}{c}
Y^{1}\left(\omega_{i}\right) \\
\vdots \\
Y^{q}\left(\omega_{i}\right)
\end{array}\right]^{T}\left[\begin{array}{ccc}
U_{1}^{1}\left(\omega_{i}\right) & \cdots & U_{1}^{q}\left(\omega_{i}\right) \\
\vdots & & \vdots \\
U_{q}^{1}\left(\omega_{i}\right) & \cdots & U_{q}^{q}\left(\omega_{i}\right)
\end{array}\right]^{-1}
$$

for $i=0, \ldots, N$. The invertibility of the $U$ matrix is a mild assumption which is satisfied if the corresponding matrix for the designed test-signal is invertible. If that is the case, then the experiments performed are said to be independent. The frequency response estimates are in themselves useful data, as they should be described by any model of the plant.

\section{FIR Model Estimation}

At this stage, we assume that we have obtained frequency response estimates at the measurement frequencies given by $\omega_{i}=\Omega_{0} \tan (\pi i / 2 L)$. Given that we can map a continuous-time system $G(s)$ into a discrete-time system $G^{d}(s)$ via the bilinear transformation,

$$
G^{d}(z)=\left.G(s)\right|_{s=\Omega_{o} \frac{z-1}{z+1}}
$$

we have that

$$
G^{d}\left(e^{\frac{j \pi i}{L}}\right)=G\left(\omega_{i}\right)
$$

at the measurement frequencies $(i=0, \ldots, N)$. Hence we have obtained frequency response estimates of $G^{d}$ at equispaced frequencies on the unit circle.

We obtain an estimate of the pulse response of $G^{d}(z)$ from an IDFT on the frequency response estimates:

$$
\hat{g}_{k}=\frac{1}{2 L} \sum_{i=-N}^{N} \hat{G}\left(\omega_{i}\right) e^{j k \pi i / L} .
$$

Next, we relate these estimates of the pulse response coefficients $\left(\hat{g}_{k}\right)$ to the true coefficients $\left(g_{k}\right)$ of the equivalent system $G^{d}(z)$ under the bilinear transformation. From the definition of this system:

$$
\begin{aligned}
G^{d}\left(e^{j \pi i / L}\right) & =\sum_{k=-\infty}^{\infty} g_{k} e^{-j \pi k i / L} \\
& =\sum_{k=0}^{2 L-1} \sum_{l=-\infty}^{\infty} g_{k+2 l L} e^{-j \pi k i / L}
\end{aligned}
$$

hence,

$$
\hat{g}_{k}=g_{k}+\sum_{l=-\infty, l \neq 0}^{\infty} g_{k+2 l L}
$$

if $L=N+1$ and $G(\infty)=G^{d}(-1)=0$.

We define $P R L_{s}$ and $P R L_{u}$ as the pulse response length of the causal and anticausal parts of $G^{d}(z)$, respectively. We use subscripts $s$ and $u$ for the pulse response lengths, because the causal and anticausal parts of the discrete-time system correspond to the stable and unstable parts of the equivalent continuous-time system. If we have chosen $L$ so that $L>P R L_{s}$ and $L>P R L_{u}$, then

$$
\hat{g}_{k} \approx\left\{\begin{array}{cc}
g_{k}, & \text { for } 1 \leq k \leq L \\
g_{k-2 L}, & \text { for } L+1 \leq k \leq 2 L
\end{array},\right.
$$

where $\hat{g}_{2 L}=\hat{g}_{0}$. Hence $\hat{g}_{k}$ for $1 \leq k \leq L$ approximates the causal part of $G^{d}(z)$ and $\hat{g}_{k}$ for $\bar{L}+\overline{1} \leq k \leq 2 L$ is diverging, approximating the anticausal part shifted by $2 L$. The pulse response lengths of the causal and anticausal parts of the system need not be the same. If, for example, the length of the anticausal pulse response is shorter than that of the causal pulse response, then fewer than $L$ elements at the tail of $\hat{g}_{k}$ will represent the anticausal part of the pulse response, and more than $L$ elements in the beginning of $\hat{g}_{k}$ will represent the causal part of the pulse response. In general, the converging part of the $\hat{g}_{k}$ sequence is dominated by the causal part of the system and the diverging part of the $\hat{g}_{k}$ sequence, if one exists, is dominated by the anticausal part of the system. A minimum requirement for $N$ is to be larger than the sum of the causal and anticausal pulse response lengths, $P R L_{u}+P R L_{s}$.

Once the pulse response coefficients $\hat{g}_{k}(k=0, \ldots, 2 L-1)$ have been identified from the frequency response estimates 
of the system, two pre-identified models are constructed, one for the stable part $\left(\hat{G}_{p-i d}^{d, s t}\right)$ and one for the unstable part $\left(\hat{G}_{p-i d}^{d, u s t}\right)$. They are defined as

$$
\hat{G}_{p-i d}^{d, s t}=\sum_{k=1}^{m_{s}} a_{k}^{s t} \hat{g}_{k} z^{-k}
$$

and

$$
\hat{G}_{p-i d}^{d, u s t}=\sum_{k=0}^{-m_{u}} a_{k}^{u s t} \hat{g}_{2 L+k} z^{-k} .
$$

where $m_{s}+m_{u}<2 L$, and $a_{k}^{s t}, a_{k}^{u s t}$ are given window functions [13]. The variables $m_{s}$ and $m_{u}$ are chosen to approximate the pulse response lengths of the stable and unstable part of the system respectively, which in turn are estimated from the identified $\hat{g}_{k}$ sequence. The variable $m_{s}$ is chosen to be the length of the converging part and $m_{u}$ the length of the diverging part of the $\hat{g}_{k}$ sequence.

The inverse bilinear transformation is then employed to obtain the continuous-time pre-identified models of the stable and unstable parts of the system from the discrete-time FIR models $\hat{G}_{p-i d}^{d, s t}$ and $\hat{G}_{p-i d}^{d, u s t}$. This transformation is,

$$
\hat{G}_{p-i d}^{s t}(s)=\left.\hat{G}_{p-i d}^{d, s t}(z)\right|_{z=\left(\Omega_{o}+s\right) /\left(\Omega_{o}-s\right)},
$$

for the stable part, and

$$
\hat{G}_{p-i d}^{u s t}(s)=\left.\hat{G}_{p-i d}^{d, u s t}(z)\right|_{z=\left(\Omega_{o}+s\right) /\left(\Omega_{o}-s\right)},
$$

for the unstable part.

Thus the pre-identified state-space model of the stable part of the continuous-time system $\hat{G}_{p-i d}^{s t}(s) \stackrel{s}{=}$ $\left(\hat{A}^{s t}, \hat{B}^{s t}, \hat{C}^{s t}, \hat{D}^{s t}\right)$ is given from the state space model of $\hat{G}_{p-i d}^{d, s t}(z) \stackrel{s}{=}\left(\hat{A}^{d, s t}, \hat{B}^{d, s t}, \hat{C}^{d, s t}, \hat{D}^{d, s t}\right)$ from:

$$
\begin{aligned}
& \hat{D}^{s t}=\hat{D}^{d, s t}-\hat{C}^{d, s t}\left(I+\hat{A}^{d, s t}\right)^{-1} \hat{B}^{d, s t}, \\
& \hat{C}^{s t}=\sqrt{2 \Omega_{o}} \hat{C}^{d, s t}\left(I+\hat{A}^{d, s t}\right)^{-1}, \\
& \hat{B}^{s t}=\sqrt{2 \Omega_{o}}\left(I+\hat{A}^{d, s t}\right)^{-1} \hat{B}^{d, s t}, \\
& \hat{A}^{s t}=\Omega_{o}\left(\hat{A}^{d, s t}-I\right)\left(I+\hat{A}^{d, s t}\right)^{-1} .
\end{aligned}
$$

Similarly we can obtain the pre-identified state-space model of the unstable part of the continuous-time system $\hat{G}_{p-i d}^{u s t}(s)$ frol. $1 \hat{G}_{p-i d}^{d, u s t}(z)$.

\section{E. Order Selection}

The Hankel singular values (h.s.v.'s) of the pre-identified state-space model are used to estimate the order of the low-order model which describes the plant. We know that the infinity norm of the error between an $m$ th order stable model $\hat{G}$ and an $r$ th order $(r<m)$ Hankel approximation $\tilde{G}_{H}$ (stable) is bounded by,

$$
\sigma_{r+1} \leq\left\|\hat{G}-\tilde{G}_{H}\right\|_{\infty} \leq \sum_{i=r+1}^{m} \sigma_{i}
$$

where the $\sigma_{i}$ 's represent the h.s.v.'s values of $\hat{G}$ ordered in decreasing order of magnitude. The lower bound is valid for any stable $r$ th order approximation. Looking at the $\sigma_{i}$ 's we can choose an order for the low-order approximation and use Hankel model reduction to obtain the stable model, $\tilde{G}_{H} \stackrel{s}{=}\left(\tilde{A}_{H}, \tilde{B}_{H}, \tilde{C}_{H}, \tilde{D}_{H}\right)$. An account of frequency weighted Hankel model reduction may be found in [20].

The stable and unstable projections of the pre-identified model are treated separately. The stable projection, $\hat{G}_{p-i, d}^{s t}$ is model reduced directly and yields $\tilde{G}_{H}^{s t}$. For the unstable part we model reduce its para-hermitian conjugate $\left(\hat{G}_{p-i d}^{u s t}\right)^{\sim}$, which is stable, to obtain the stable approximation $\left(\tilde{G}_{H}^{u s t}\right)^{\sim}$. Then $\tilde{G}_{H}^{u s t}$ is the reduced-order model for $\hat{G}_{p-i d}^{u s t}$ and the complete Hankel model is,

$$
\tilde{G}_{H}=\tilde{G}_{H}^{s t}+\tilde{G}_{H}^{u s t} \stackrel{s}{=}\left(\tilde{A}_{H}, \tilde{B}_{H}, \tilde{C}_{H}, \tilde{D}_{H}\right)
$$

\section{F. The Identified Model}

To obtain the final identified model we refine the loworder IIR model derived above using Chebycheff approximation [17],[19]. A general method for MIMO systems can be found in [21],[18], but here we concentrate on the MISO case as the Tokamak response we are considering has a relatively large number of inputs and outputs. Approximating the MIMO system directly would have led to a prohibitive computational problem.

First we present the "zero-only" tuning algorithm $[17],[18],[19]$, in which we tune the zero polynomial of the identified model $\tilde{G}_{I}(s)$, such that,

$$
\tilde{G}_{I}(s)=\arg \min _{\tilde{G}(s)} \max _{s_{i}=j \omega_{i}} \bar{\sigma}\left[\left(\hat{G}\left(s_{i}\right)-\tilde{G}\left(s_{i}\right)\right) W\left(s_{i}\right)\right]
$$

i.e. the infinity norm of the weighted error between the identified model and the frequency response estimates is minimised; $W(s)$ is a given weighting function. The pole polynomial is fixed, and derived from the $r$ th order Hankel model (Section 2.5). The identified model can be written as,

$$
\tilde{G}_{I}(s)=\frac{\tilde{N}_{I}(s)}{\tilde{d}_{H}(s)}=\frac{\sum_{l=0}^{n} \mathbf{b}_{\mathbf{l}}{ }^{T} s^{l}}{\tilde{d}_{H}(s)}, \quad n \leq r,
$$

in which the $\mathbf{b}_{\mathbf{l}}$ 's are $q$-dimensional column vectors and $\tilde{d}_{H}(s)$ is the Hankel pole-polynomial.

The zero-only approximation problem (2) is solved with respect to the $\mathbf{b}_{\mathbf{l}}$ 's, via the following minimax problem,

$$
\begin{aligned}
& \begin{array}{ll}
\text { minimise } & \epsilon \\
\text { subject to } & \epsilon+\Re\left\{\frac{\sum_{l=0}^{n} \mathbf{b}_{\mathbf{l}}{ }^{T} s_{i}^{l}}{\tilde{d}_{H}\left(s_{i}\right)} W\left(s_{i}\right) \mathbf{v}^{\mathbf{i}}\right\}
\end{array} \\
& \geq \Re\left\{\hat{G}\left(s_{i}\right) W\left(s_{i}\right) \mathbf{v}^{\mathbf{i}}\right\}
\end{aligned}
$$

for all $i=0, \ldots, N-1$ and all $\left\|\mathbf{v}^{\mathbf{i}}\right\|=1$ with $\epsilon>0$. This minimax problem is converted into a linear program by writing it in the equivalent form: 


$$
\begin{aligned}
& \text { minimise } \epsilon \\
& \text { subject to } \\
& \begin{array}{r}
{\left[\begin{array}{c}
\mathbf{b}_{\mathbf{0}} \\
\vdots \\
\mathbf{b}_{\mathbf{n}} \\
\epsilon
\end{array}\right]^{T} \Re\left\{\left[\begin{array}{c}
W\left(s_{i}\right) \mathbf{v}^{\mathbf{i}} \\
\vdots \\
W\left(s_{i}\right) \mathbf{v}^{\mathbf{i}} s_{i}^{n} \\
1
\end{array}\right]\right\}} \\
\geq \Re\left\{\hat{G}\left(s_{i}\right) W\left(s_{i}\right) \mathbf{v}^{\mathbf{i}}\right\} .
\end{array}
\end{aligned}
$$

Because this is a convex problem, the solution found is guaranteed to be globally optimal [21, Characterisation theorem]. The algorithm solving the linear program (3) is also described in [21],[18].

It is also possible to use an $\mathcal{L}^{\infty}$ variant of Levy's method $[18],[22]$ to tune both the poles and the zeros of the MISO identified model $\tilde{G}_{I}(s)$. This is the "zero-and-pole" identification problem: find $\mathbf{b}_{\mathbf{1}},(l=0, \ldots, n)$ and $a_{k},(k=$ $1, \ldots, r)$ such that

$$
\tilde{G}_{I}(s)=\frac{\tilde{N}_{I}(s)}{1+\tilde{\phi}_{I}(s)}=\frac{\sum_{l=0}^{n} \mathbf{b}_{\mathbf{l}}^{T} s^{l}}{1+\sum_{k=1}^{r} a_{k} s^{k}}, \quad n \leq r+1
$$

minimises the infinity-norm of the identification error,

$$
\tilde{N}_{I}, \tilde{\phi}_{I}=\min _{\tilde{N}_{I}, \tilde{\phi}_{I}} \max _{i=0, \ldots, N-1} \bar{\sigma}\left[\left(\hat{G}\left(s_{i}\right)-\tilde{G}_{I}\left(s_{i}\right)\right) W\left(s_{i}\right)\right] .
$$

This approximation problem is solved by an iterative process:

$$
\begin{gathered}
\tilde{N}_{I}^{m+1}, \tilde{\phi}_{I}^{m+1}= \\
\arg \min _{N, \phi} \max _{i} \bar{\sigma}\left[\left(\hat{G}\left(s_{i}\right)\left(1+\phi\left(s_{i}\right)\right)-N\left(s_{i}\right)\right) w^{m}\left(s_{i}\right)\right],
\end{gathered}
$$

where at each iteration we update $w^{m}(s)=W\left(s_{i}\right) /(1+$ $\left.\tilde{\phi}_{I}^{m}(s)\right)$ starting with $w^{1}(s)=W\left(s_{i}\right) /\left(1+\hat{\phi}^{H}(s)\right)$ where $\hat{\phi}^{H}(s)$ given from $\tilde{G}_{H}(s)$. Once this process has converged, after $M$ iterations say, the resulting model is given by

$$
\tilde{G}_{I}(s)=\frac{\tilde{N}_{I}^{M}(s)}{1+\tilde{\phi}_{I}^{M}(s)} .
$$

The linear program which solves the $m$ th iteration is,

minimise $\quad \epsilon$
subject to

$$
\begin{gathered}
{\left[\begin{array}{c}
\mathbf{b}_{\mathbf{0}} \\
\vdots \\
\mathbf{b}_{\mathbf{n}} \\
-a_{1} \\
\vdots \\
-a_{r} \\
\epsilon
\end{array}\right]^{T} \Re\left\{\left[\begin{array}{c}
w^{m}\left(s_{i}\right) \mathbf{v}^{\mathbf{i}} \\
\vdots \\
w^{m}\left(s_{i}\right) \mathbf{v}^{\mathbf{i}} s_{i}^{n} \\
\\
\hat{G}\left(s_{i}\right) w^{m}\left(s_{i}\right) \mathbf{v}^{\mathbf{i}}\left[\begin{array}{c}
s_{i} \\
\vdots \\
s_{i}^{r}
\end{array}\right] \\
1
\end{array}\right]\right\}} \\
\geq \Re\left\{\hat{G}\left(s_{i}\right) w^{m}\left(s_{i}\right) \mathbf{v}^{\mathbf{i}}\right\} .
\end{gathered}
$$

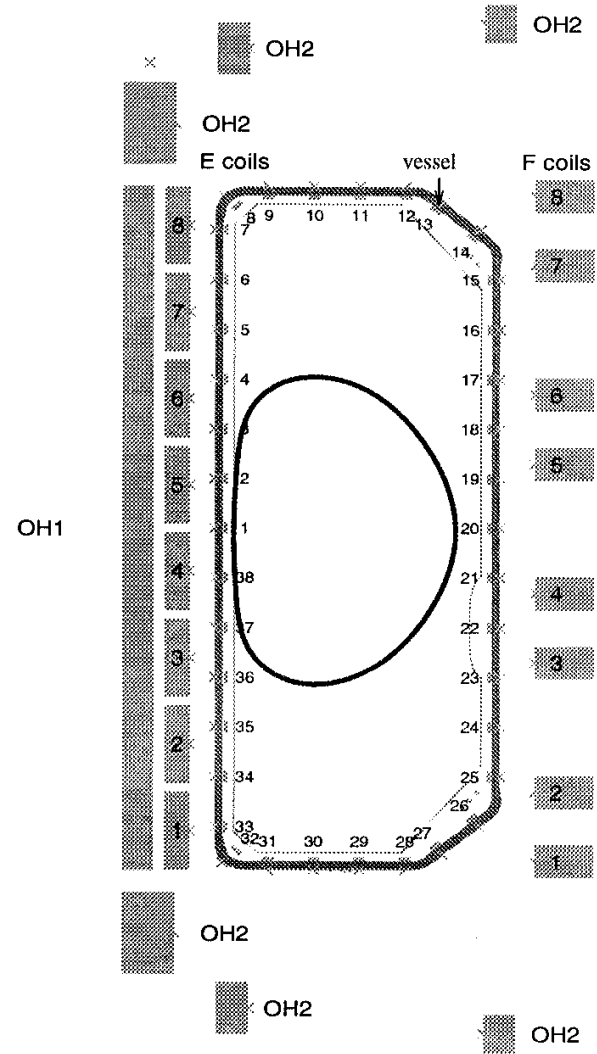

Fig. 2

TCV CROSS-SECTION SHOWING THE POSITION OF THE POLOIDAL FIELD ( $\mathrm{E}, \mathrm{F}$ and $\mathrm{OH}$ ) COILs. The contour shows the CROSS-SECTION OF THE PLASMA USED IN THESE EXPERIMENTS.

The zero-and-pole approximation problem is non-convex and the iterations (5) need not converge. However, if they converge then the solution of (6) is the global optimum of the zero-and-pole approximation problem (4), [21].

Furthermore, it has to be checked, a posteriori, whether the identified model obtained from zero-and-pole tuning has a number of unstable poles compatible with the Hankel singular values of the FIR model [21]. This is because the $a_{k}$ 's are not constrained to preserve a stability property.

\section{TCV System IDENTIFICATION}

\section{A. The TCV Tokamak}

TCV is a Tokamak which is ideally suited to a plasma control and modelling study as it possesses a relatively large number of independently driven poloidal field coils (Figure 2). This feature lends an adaptability not available in other machines. Referring to Figure 2, these coils comprise an inner set of $8 \mathrm{E}$ coils, an outer set of $8 \mathrm{~F}$ coils, a single large ohmic coil (OH1) and a set of smaller ohmic coils, connected in series, which are all labelled OH2. The ohmic coils are used for inductively driving the plasma current, while the $\mathrm{E}$ and $\mathrm{F}$ coils are used for plasma shaping and positioning. Rather than using the $\mathrm{E}$ and $\mathrm{F}$ coils independently they were paired symmetrically with respect to 
the horizontal mid-plane to form the pairs E1-E8, E2-E7, E3-E6, E4-E5, F1-F8, F2-F7, F3-F6 and F4-F5. The coilpairs could then be used in two modes, symmetric (where the coil-pairs receive the same voltage) and antisymmetric (where the coil-pairs receive voltages of the same magnitude but of opposite sign). The modelling of TCV was performed in two stages, the identification of a symmetric plasma response and the identification of an antisymmetric plasma response. Linking the coils in this fashion is useful as it allows a decoupling of the plasma parameters. In particular the plasma vertical position is the only parameter excited in the antisymmetric experiments. (Though this is only true for plasmas which are themselves symmetric about the mid-plane. It is true for the situations examined here). This decoupling occurs because the antisymmetric experiments are the only ones which can produce a net radial magnetic field at the mid-plane. Another potentially useful consequence of performing the experiment in this way is that we can reduce the signal to noise ratio of the data, as each model input comprises two independent coil voltage inputs, each with similar characteristics.

In these experiments there were ten possible inputs, the eight coil-pairs and the two ohmic coils, but after performing both the symmetric and antisymmetric experiments we would have the data required to describe the action of the 18 independent coils of the full system. The linking of the ohmic coils meant that they could only contribute to the symmetric experiments, so the symmetric and antisymmetric experiments had 10 and 8 inputs respectively. The control parameters (cp) of interest are 6 plasma properties: the inner and outer curvature of the plasma boundary (Figure $2)$, two estimates of the plasma radial position $\left(\Psi_{r}, \mathrm{P}_{\text {vert }}\right)$, the plasma current $\left(I_{p}\right.$, Figure 1$)$ and the product of $I_{p}$ with the vertical plasma position $\left(Z I_{p}\right)$. A more detailed description of these parameters is given in [6]. In these experiments the' plasma shape was chosen such that the system was only mildly unstable (low elongation).

Our aim was to obtain a full MIMO model of the 18 coil inputs to the 6 outputs. In practice this was achieved by combining SISO and in some cases MISO models. This was due to the particular challenges involved in identifying TCV.

A simple PID controller, which acts through all coils, was always used to control the plasma position. A block diagram of the TCV Tokamak including its controller and power supply loop is shown in Figure 3 . The function of the power supply loop is to constrain the current in the coils. We consider the power supply to be part of the open-loop TCV system and thus seek to identify a model for $G(s)$ in Figure 3. Measurements of the voltage commands to the power supplies $(u)$ and of the control parameters $(c p)$ are always available.

\section{B. The Identification Experiments}

As described earlier, two separate sets of experiments were performed, a symmetric set and an antisymmetric set. For each experiment in a set, a different coil-input was excited, as described in Section 2.1, but it should be

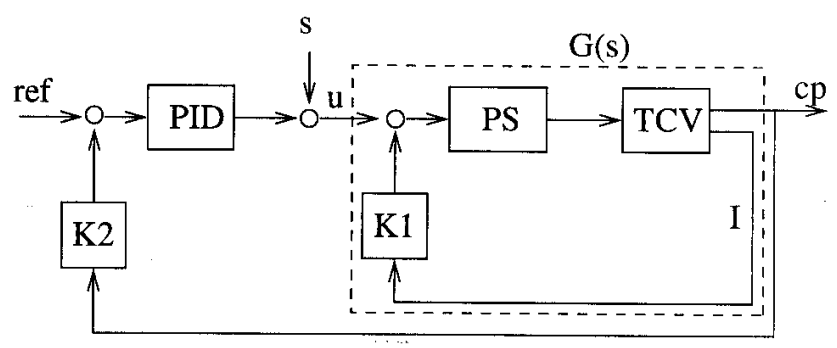

Fig. 3

The TCV experiment Layout. PID is a SIMPLE PID CONTROller USED TO STABILISE TCV, PS IS THE POWER SUPPLY AND $c p$ DENOTES THE CONTROL PARAMETERS. $G(s)$ IS THE TRANSFER FUNCTION TO BE IDENTIFIED, WHICH INCLUDES TCV AND THE POWER SUPPLY LOOP.

noted that whereas the test signal was only applied to one coil-input at a time, the controller used all of the inputs. The aim of the identification procedure was to model the relationship between the input of the power supply loop to the control parameters.

The TCV excitation signal was designed with 29 sine waves spanning the frequency range $20 \mathrm{rad} / \mathrm{sec}$ to $3 k r a d / s e c$. The experimental conditions define the range of frequencies that we can design in the excitation signal (see Section 2.1). On TCV a single experiment is limited to about $0.5 \mathrm{sec}$. Assuming $50 \mathrm{msec}$ as an estimate of the time it takes for the transients to decay, we are left with $0.45 \mathrm{sec}$ of useful identification data. The period of the slowest sinewave in the excitation signal was designed to be $0.3 \sec \left(=2 \pi / \omega_{1}\right)$ and this should be comfortably resolved during such an experiment. The highest excitation frequency was designed to be well below half the sampling frequency, (sampling is at $5 k H z$ on TCV) and also well above the TCV open-loop bandwidth, (which was known from previous TCV operation).

This excitation signal $s(t)$ (injected at point $s$ in Figure 3) was defined by

$$
s(t)=\sum_{i=1}^{29} k_{i} M \cos \left(\omega_{i} t+\phi_{i}\right)
$$

where the phases $\left(\phi_{i}\right)$ were chosen as described earlier to minimise the overall amplitude of the signal and the frequencies $\left(\omega_{i}\right)$ were defined by

$$
\omega_{i}=430 \tan \left(\frac{\pi i}{64}\right) .
$$

The weighting factor $k_{i}$ was chosen to be

$$
k_{i}=\left[\begin{array}{lllllll}
1 & \cdots & 1 & 1.4 & 3 & 4 & 4
\end{array}\right] \text {, }
$$

where larger weighting factors were given to the higher frequency sine waves in order to compensate for the lower gain of the closed loop of TCV at these frequencies. The value of $M$ was chosen such that the voltages applied on the PF coils lay well within $80 \%$ of the power supply limits. These 

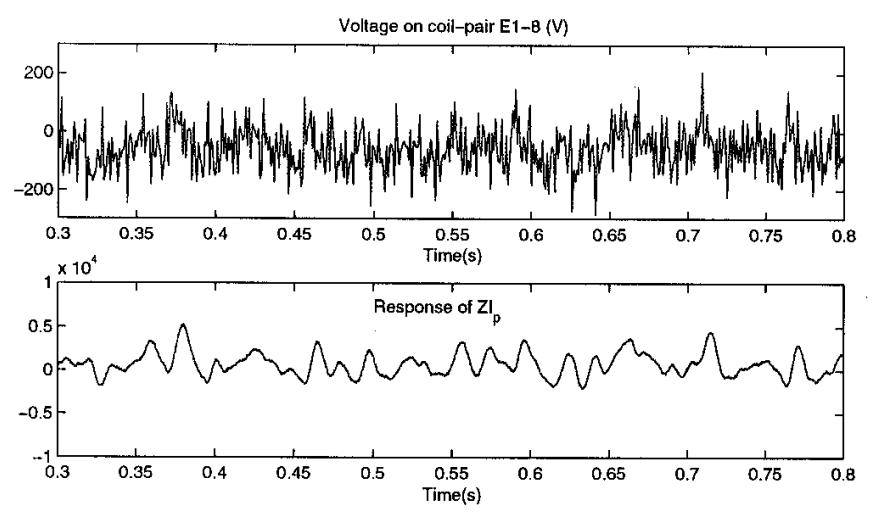

Fig. 4

Data from EXPERIMENTAL SHOT NUMber 13333. The inPUt to COIL PAIR E1-8 is ILLUSTRATED aLONG WITH THE RESPONSE IN THE CONTROL PARAMETER $Z I_{p}$.

limits are $650 \mathrm{~V}$ for the E-coils, $1250 \mathrm{~V}$ for the F-coils and $1400 \mathrm{~V}$ for the $\mathrm{OH}$ coils. The same excitation signal (allowing for differt nt $M$ ) was used for all experiments.

All the data was subject to drift and offset and these phenomena were removed before analysis: The use of the present PID controller implied that it would be impossible to resolve a DC input. Typical input and output data are shown in Figure 4.

\section{Identified Model \\ C.1 Antisymmetric Response}

As described in Section 3.1, $Z I_{p}$ is the only control parameter which needs to be modelled for the antisymmetric data set, as it is the only output which can be driven. The experimental data from the 8 experiments was processed as detailed in Sections 2.2-2.4 to produce the pulse response of an equivalent discrete-time system (Figure 5). Based on this data we selected the length of the converging part of the pulse response $\left(m_{s}\right)$ to be 35 and the length of the diverging part $\left(m_{v i}\right)$ to be 15 . Once defined, these converging and diverging pulse responses were used to approximate FIR high-order models of the stable and unstable parts of the ZIP response respectively (Section 2.4). The order of these FIR models was estimated by examination of the Hankel singular values of their weighted Hankel approximations (Section 2.5). These values are shown in Figure 6 and it may be seen that the order of the unstable system is dominated by 1 state, while the stable part may be approximated by a 3 state model. The single unstable mode corresponds to the existence of the vertical plasma instability and we know from the underlying physics that there should be only one unstable mode. The final identified model was produced using the zero-and-pole Chebycheff algorithm (Section 2.6). During this calculation, and for the frequencies below $800 \mathrm{rad} / \mathrm{sec}$, the error on each frequency response estimate was weighted by the inverse of its amplitude. Above $800 \mathrm{rad} / \mathrm{sec}$ the weight amplitude was kept
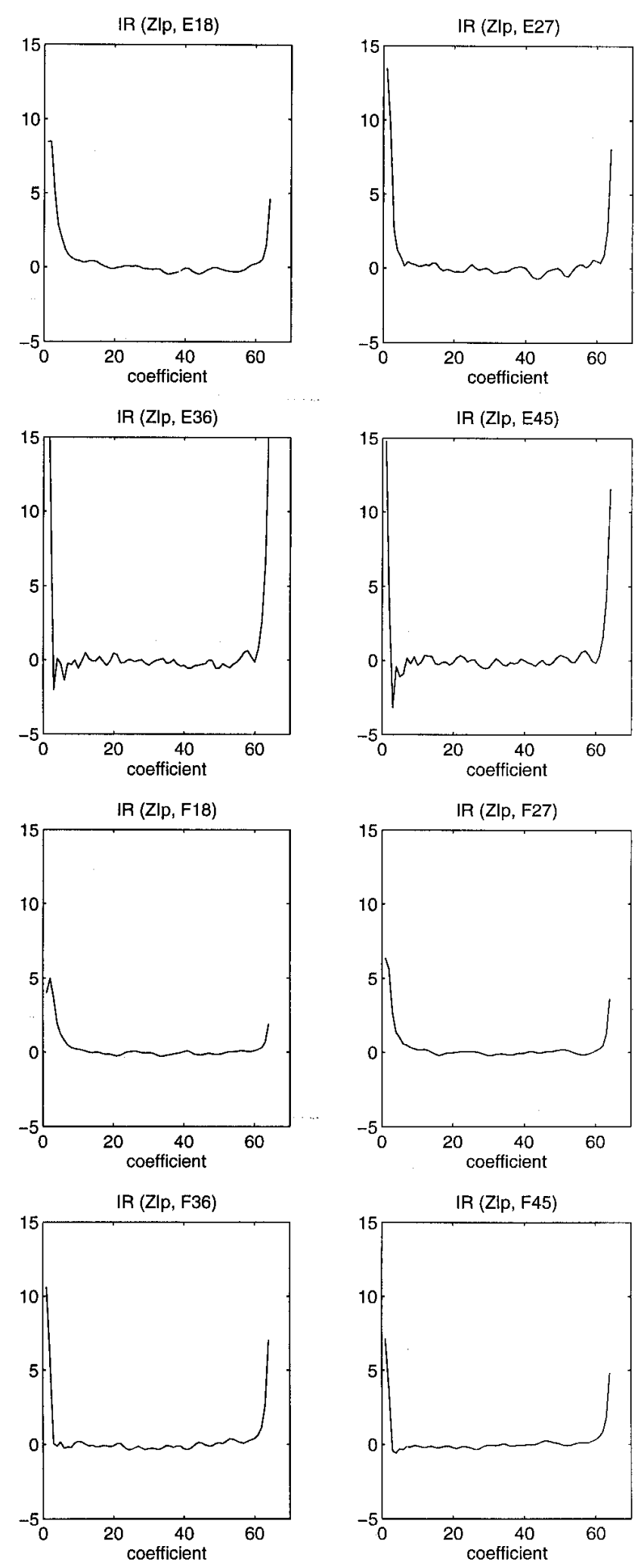

Fig. 5

ESTIMATED PULSE RESPONSE OF THE EQUIVALENT DISCRETE-TTME SYSTEM OF THE $Z I_{p}$ RESPONSE TO THE EIGHT PF COIL-PAIRS. The $\mathrm{X}$-AXIS IS AN INDEX TO THE PULSE RESPONSE COEFFICIENTS. 

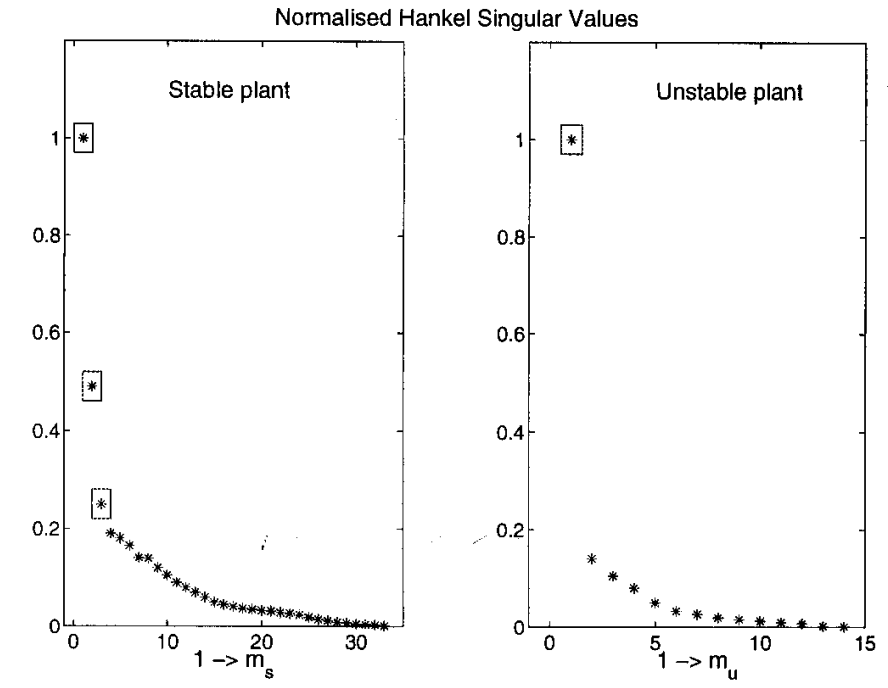

Fig. 6

Normalised Hankel singular values from weighted Hankel APPROXIMATION ON THE HIGH-ORDER STABLE AND UNSTABLE FIR MODEL. BOXED VALUES ARE CONSIDERED DOMINANT.

constant. This weighting was chosen to maintain an accurate approximation to the frequency response estimates up to $800 \mathrm{rad} / \mathrm{sec}$. Above $800 \mathrm{rad} / \mathrm{sec}$ however, the accuracy of the approximation is less important.

The final 4th order model is described by,

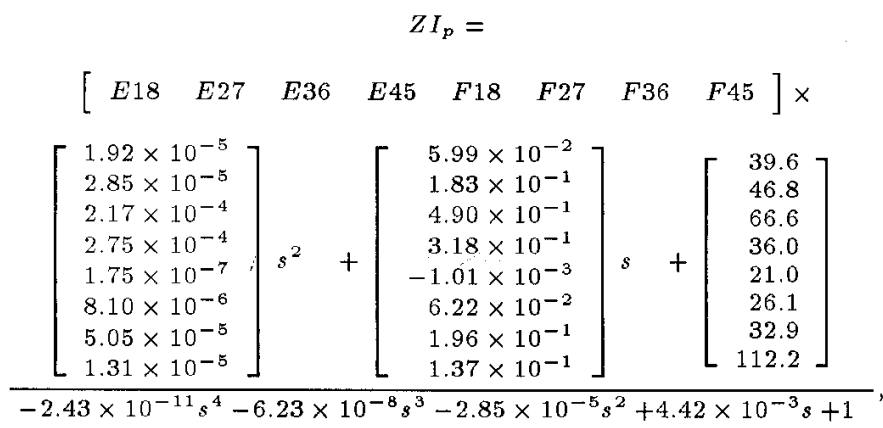

where $E 18$ i.s an the abbreviation for $E 1-E 8$, etc. This model includes the effective resistance of the power supply loop but a phase delay of $0.7 \mathrm{~ms}$ must be added to produce the Figures shown. This phase delay is a reasonable value for the devices used.

The antisymmetric identified model was compared with a physics model which assumes that the plasma current behaves as a filament moving in plasma averaged fields. This model also assumes an experimentally observed flux conservation law and has plasma shape correction functions [5]. In predictions of vertical behaviour it is similar to the Rigid Current Displacement Model (RCDM, [3]). Their comparison is illustrated in Figures 7 and 8 . It can be seen that the identified model is in excellent agreement with the RCDM and the predictions of the growth rate of the vertical instability are very close $\left(213 \mathrm{sec}^{-1}\right.$ identified vs $212 \mathrm{sec}^{-1}$ Filament or $209 \mathrm{sec}^{-1}$ RCDM). This is an encouraging cross validation of the identified and the physics

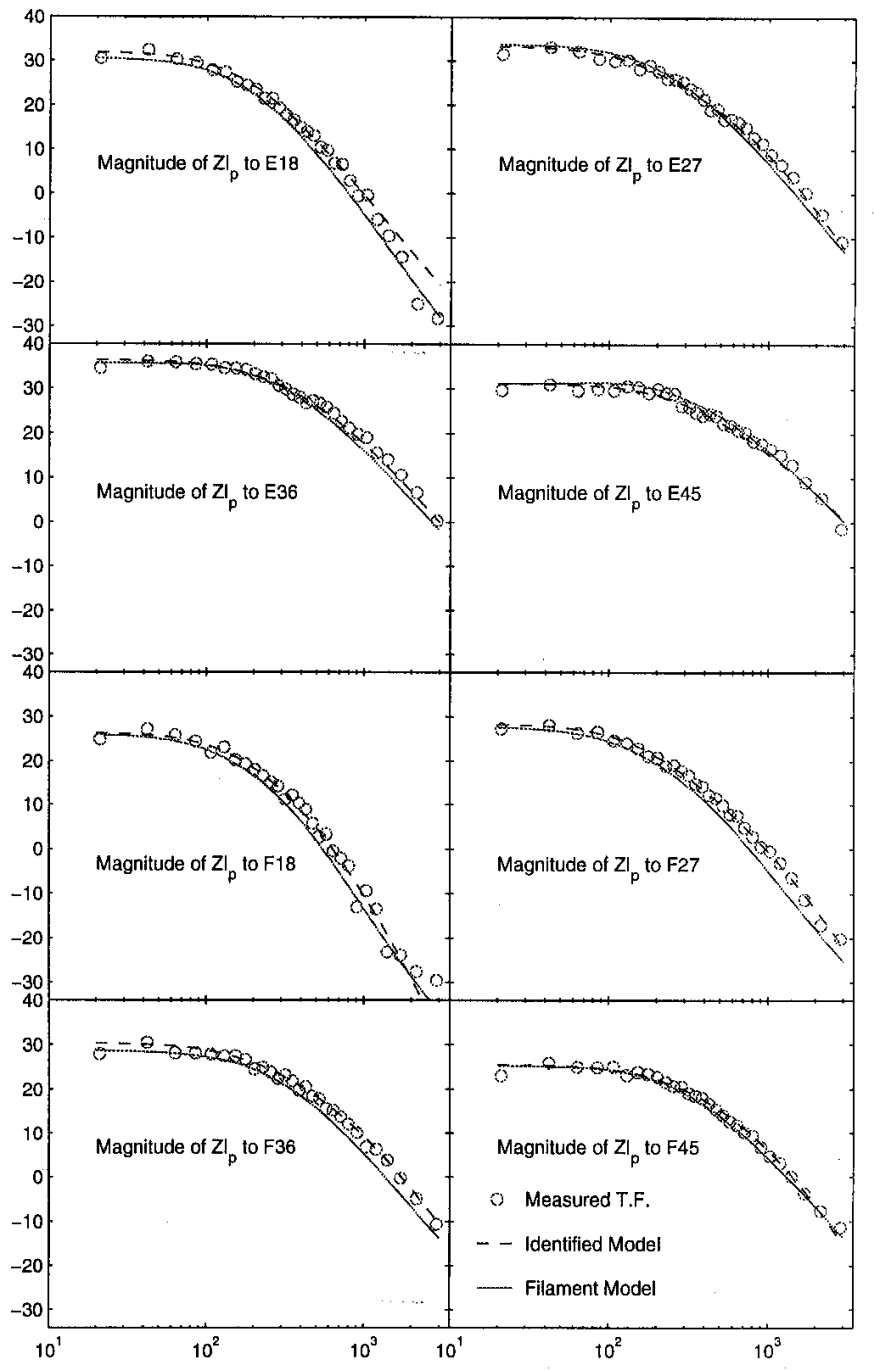

Fig. 7

AMPLITUdE RESPONSE OF THE IDENTIFIED MODEL (DASHED), FREQUENCY RESPONSE ESTIMATES (CIRCLES) AND FilAMENT MODEL (SOLID). AMPLITUDE AXIS MARKED IN $d B$, FREQUENCY IN $\mathrm{rad} / \mathrm{sec}$.

based models. The identified model can also be used to look for inconsistency in the physics based models and in this case we can see room for improvement in the amplitude responses of the E1-E8 and F3-F6 coil pairs and the phase of F1-F8.

\section{C.2 Symmetric Response}

The symmetric behaviour of TCV was found to be more complex than the asymmetric behaviour. This can be understood from the fact that the symmetric response of the plasma, unlike the asymmetric response, is not dominated by any single mode. However low order identified models describing the response of the control parameters to voltages on the PF coils could be produced. The order of the models would seem to imply the existence of an underlying simplicity of behaviour of the Tokamak (a concept developed in [6]). It was found that there was little overlap of 


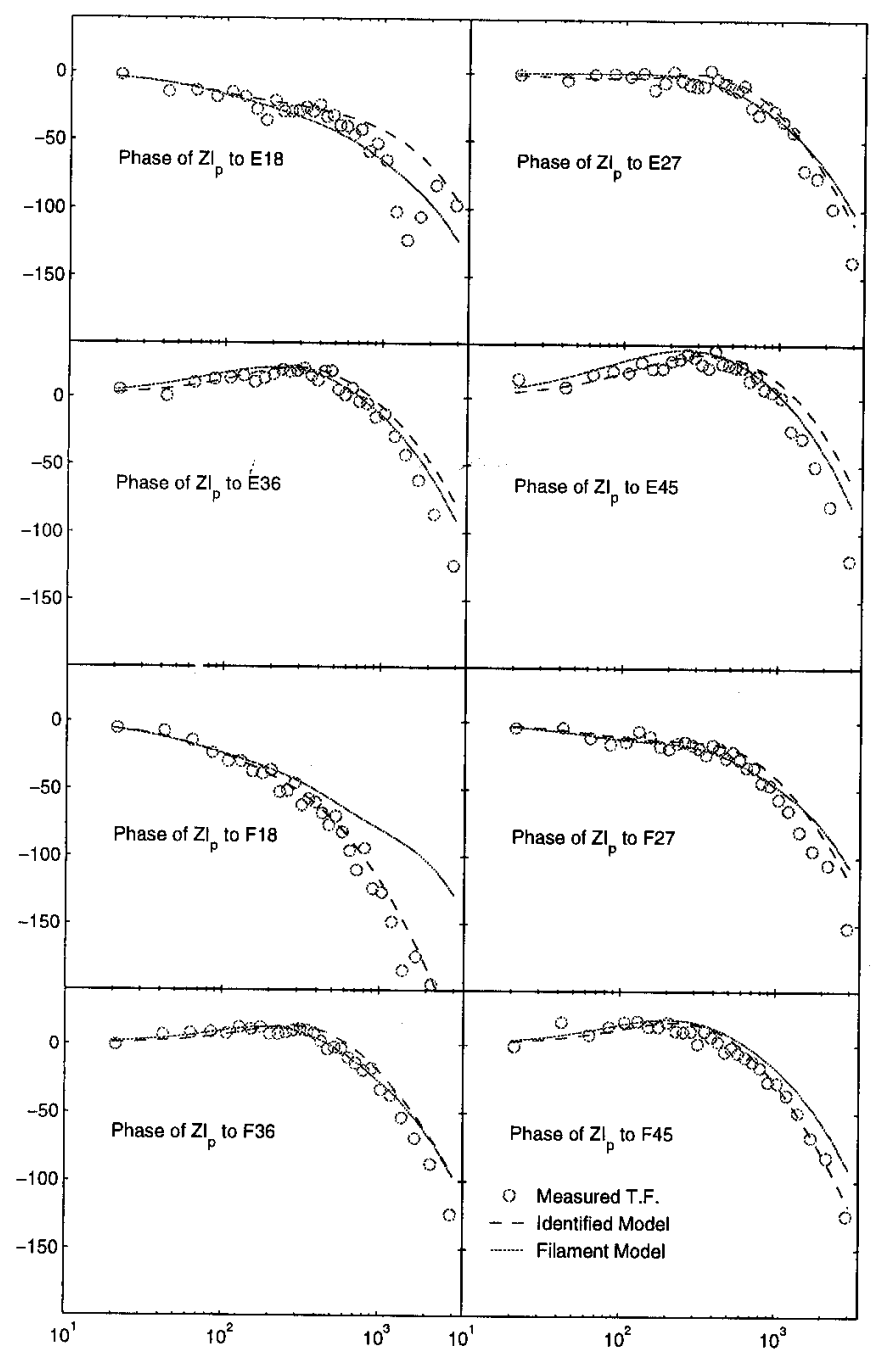

Fig. 8

Phase Response of IDENTIFIEd MOdel (DASHed), FREQUency Response estimates (Circles) and Filament model (SOlid).

Phase aXIS MARIEE IN DEGREes, FREQUENCY IN $\mathrm{rad} / \mathrm{sec}$.

the modes describing the various coils, and so SISO and in some cases $1 \times 2$ MISO models were created.

The transient response of the tokamak in this configuration was found to be longer than our 50 msec estimate. As the experiment time is constrained to $0.5 \mathrm{sec}$, the useful data was reduced to almost $0.4 \mathrm{sec}$ which was not sufficient to resolve the lowest frequency accurately. Also in one or two cases the data was not of sufficient quality to create a valid model from the basic algorithms. However by comparison with the models of the nearest coils we were able to approximate the unresolved model, and in this way complete the full identification process. An example of such data is the E2-E7 response in 9, Here the ID model was produced via a small perturbation of the E1-E8 model.

Figures 9 and 10 show the identified response of the control parameter $\Psi_{r}$ (one of the estimates of the plasma radial position) to the voltages on the coils. These results are typ-

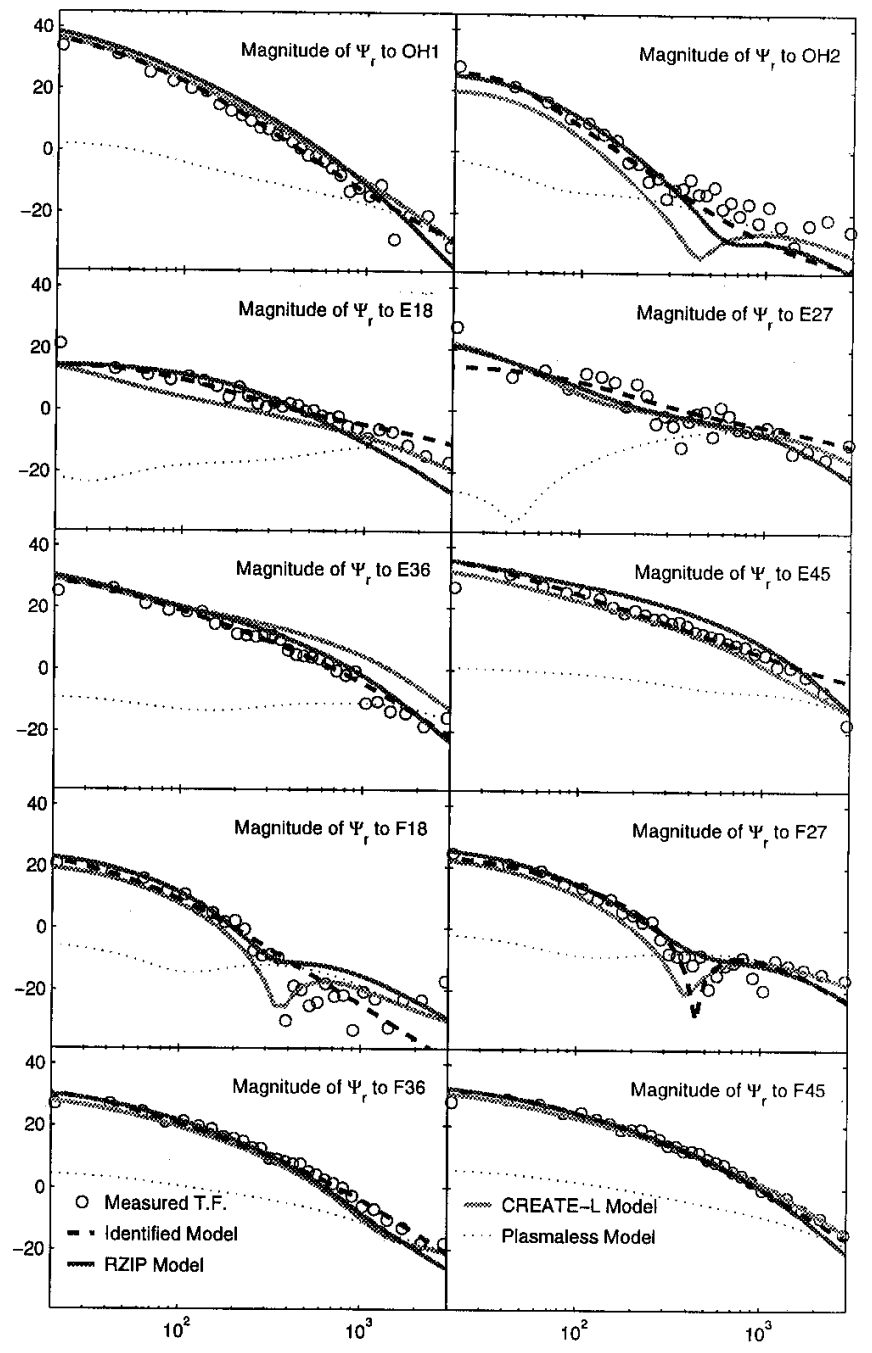

Fig. 9

AMPLITUDE RESPONSE OF IDENTIFIED MODEL (DASHED), FREQUENCY RESPONSE ESTIMATES (CIRCLES), TWO PHYSICS PLASMA MODELS

(SOLID) AND A PLASMALESS PHYSICS MODEL (DOTTED). AMPLITUdE AXIS MARKED IN $d B$, FREQUENCY IN $\mathrm{rad} / \mathrm{sec}$.

ical of the other outputs. The response is modelled with 10 SISO models, mostly of 3rd order. As can be seen in these figures the frequency response estimates at the lowest frequency are relatively inaccurate. Consequently, they were ignored when obtaining the final identified model.

The model of TCV which is currently used to tune the PID controller, is the low frequency response of the 'plasmaless' model. This is a model from the coil voltages to the magnetic diagnostics in the absence of the plasma and it represents the effects of the surrounding metallic structures. It is shown in Figures 9 and 10 as a dotted line and can be seen to be a relatively poor model. We can therefore expect a significant improvement when we use the identified model for controller design. Two other physics based models (RZIP and CREATE-L) are plotted on the graphs and are detailed in Reference [6]. These models predict slightly different behaviours and the identified model is used as a 


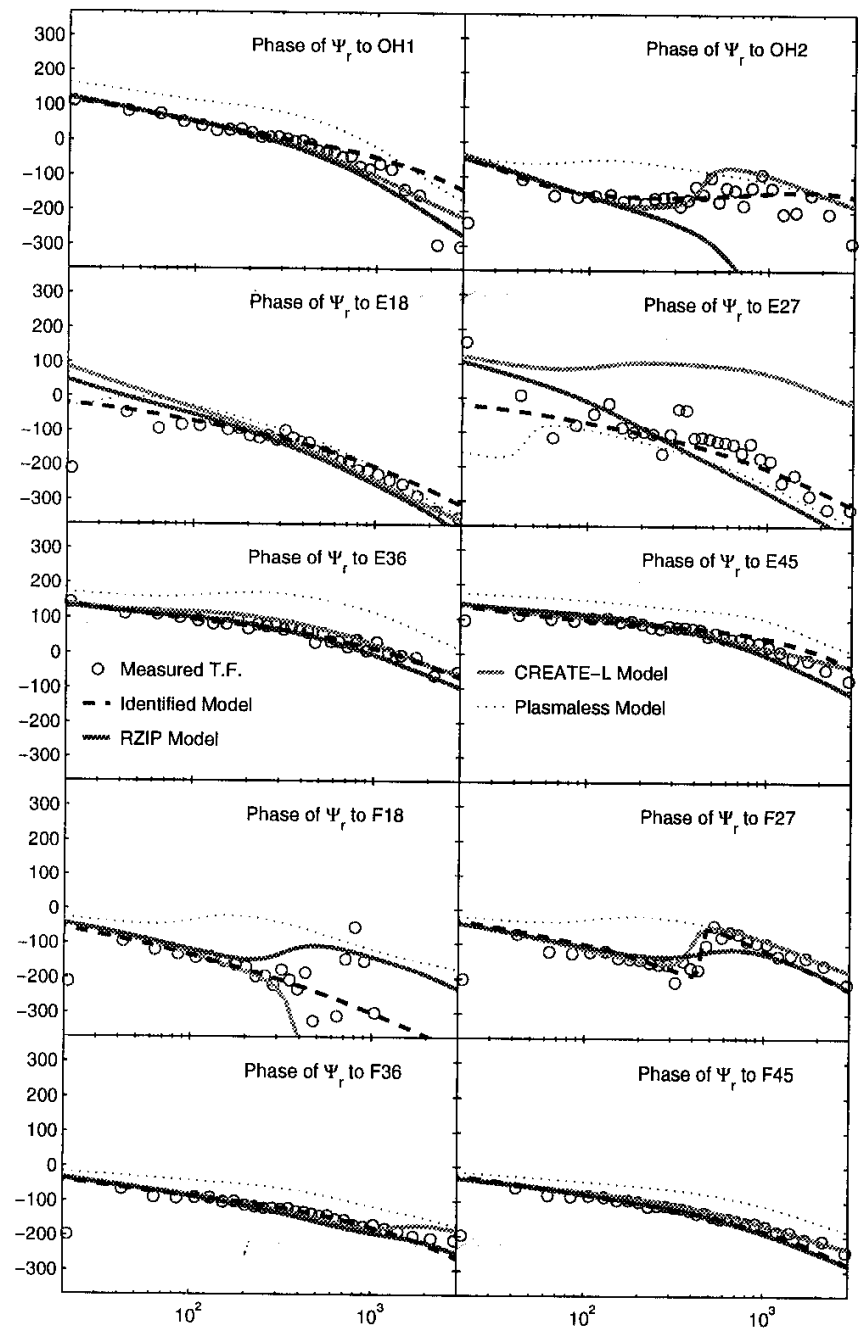

Fig. 10

Phase Resp Jnse of IDENTIFIEd MOdel (DASHED), FREQUency RESPONSE ESTIMATES (CIRCLES) TWO PHYSICS MODELS (SOLID) AND A Plasmaless physics model (DOTTed). Phase axis Marked in DEGREES, FREQUENCY IN $\mathrm{rad} / \mathrm{sec}$.

check on their physics assumptions.

\section{Conclusions and Further Work}

We have presented an application of an $\mathcal{H}^{\infty}$ identification technique for MISO, unstable and continuous-time systems [17],[18], to a plasma physics experiment. In particular, this procedure has been successfully applied to the TCV Tokamak, and used to create a model of the open-loop response of the plasma to voltages applied to the poloidal field coils. The TCV Tokamak was a demanding test for the method as it exhibits complex dynamics, including one unstable pole, and has 6 outputs and 18 inputs. The value of this identified model is twofold: firstly, it may be used to verify, or invalidate an assortment of physics based models, and secondly it may be used in its own right as the basis of a controller design exercise.

For the modelling of the vertical position of the plasma, the identified model was found to be in excellent agreement with an available physics model. We have seen in Figures 7 and 8, that the measured and predicted frequency responses are almost identical. Furthermore, we predicted the presence of a single unstable pole and obtained close correlation between the identified and predicted growth rates.

The symmetric response of TCV was found to be more complicated than the asymmetric response, but the identification procedure was still able to produce viable low order models of the system. Thus the total (symmetric and asymmetric) response of one type of TCV plasma has been completed. The symmetric experiments could be improved (the unexpected persistence of the transient could be allowed for) but the current data and process seem satisfactory.

In our future work we plan to design and test an improved controller using the identified models obtained in this work. We will also test the methodology using a more unstable plasma.

\section{Acknowledgements}

We freely acknowledge the help of the following people with this work. R. Albanese and F. Villone for the CREATE-L model [7] and I. Banyopadhyay for help on the RZIP model. We also thank the following for assistance with funding, Euratom mobility funding for A.C. (3082), Fonds national Suisse de la recherche scientifique and the E.P.S.R.C.

\section{REFERENCES}

[1] J. B. Lister et. al., "The control of TCV plasmas," Fusion Technology, vol. 32(3), pp. 321-373, 1997.

[2] R. Albanese, E. Coccorese, and G. Rubinacci, "Plasma modelling for the control of vertical instabilities in tokamaks.," $\mathrm{Nu}$ clear Fusion, vol. 29, no. 6, p. 1013, 1989.

[3] J. B. Lister, Y. Martin, and J.-M. Moret, "On locating the poloidal field coils for tokamak vertical position control," $N_{u-}$ clear Fusion, vol. 36, pp. 1547-1560, 1996.

[4] C. E. Kessel, M. A. Firestone, and R. W. Conn, "Linear optimal control of tokamak fusion devices," Fusion Technology, vol. 17(5), pp. 391-414, 1990.

[5] J. P. Wainwright, D. Copsey, D. J. N. Limebeer, M. G. Haines, and A. Portone, "Extensions to single filament modelling of a tokamak plasma," Nuclear Fusion, vol. 37(12), pp. 1679-1690, 1997.

[6] A. Coutlis, I. Bandyopapadhyay, J. B. Lister, P. Vyas, R. Albanese, D. J. N. Limebeer, F. Villone, and J. P. Wainwright, "Measurements of the open loop plasma equilibrium response in TCV," Nuclear Fusion, vol. 39(5), pp. 663-685, 1999.

[7] F. Villone, P. Vyas, J. B. Lister, and R. Albanese, "Comparison of the CREATE-L plasma response model with TCV limited discharges," Nuclear Fusion, vol. 37, pp. 1395-1410, 1997.

[8] F. Hofmann, M. J. Dutch, D. J. Ward, M. Anton, I. Furno, J. B. Lister, and J.-M. Moret, "Vertical instability in TCV: Comparison of experimental and theoretical growth rates," Nuclear Fusion, vol. 37, pp. 681-687, 1997.

[9] P. M. Mäkilä, J. R. Partington, and T. K. Gustafsson, "Worstcase control-relevant identification," Automatica, vol. 31, pp. 1799-1819, 1995.

[10] B. Ninness and G. C. Goodwin, "Estimation of model quality," Automatica, vol. 31, pp. 1771-1797, 1995.

[11] P. M. J. V. Den Hof and R. J. P. Schrama, "Identification and control - closed loop issues," Automatica, vol. 31, pp. 1751-1770, 1995. 
[12] J. H. Friedman and P. P. . Khargonekar, "Application of identification in $\mathcal{H}^{\infty}$ to lightly damped systems:two case studies.," IEEE Transactions on Control Systems Technology, vol. 3, pp. 279-290, 1995.

[13] G. Gu and P. P. Khargonekar, "Linear and nonlinear algorithms for identification in $\mathcal{H}^{\infty}$ with error bounds," IEEE Trans. Autom. Control, vol. 37, pp. 953-963, 1992.

[14] A. J. Helmicki, C. A. Jacobson, and C. N. Nett, "Control oriented system identification: A worst-case deterministic approach in $\mathcal{H}^{\infty}$," IEEE Trans. Autom. Control, vol. 36, pp. 1163-1176, 1991.

[15] A. J. Helmicki, C. A. Jacobson, and C. N. Nett, "Worst-case deterministic identification in $\mathcal{H}^{\infty}$ : The continuous-time case," IEEE Trans. Autom. Control, vol. 37, pp. 604-610, 1992.

[16] N. P. Rubin and D. J. N. Limebeer, " $\mathcal{H} \infty$ identification for robust control," in Proc. Am. Control Conf., pp. 2040-2044, 1994.

[17] A. Coutlis and D. J. N. Limebeer, "Control oriented system identification for MIMO continuous and discrete-time systems," Proc. 35th Conf. on Decision and Control, 1996. Kobe, JAPAN.

[18] A. Coutlis and D. J. N. Limebeer, "Chebycheff approximation in system identification and model reduction," Automatica, vol. 32, 1997.

[19] A. Coutlis, J. B. Lister, P. Vyas, D. J. N. Limebeer, and J. P. Wainwright, "An $\mathcal{H}^{\infty}$ identification algorithm applied to tokamak modelling," in IEEE 36 th Conference on Decision and Control, (San Diego, CA), 1997.

[20] M. Green and D. J. N. Limebeer, Linear Robust Control. Prentice Hall, 1995.

[21] A. Coutlis, System Identification for MIMO Discrete and Continuous-Time Systems. PhD thesis, Department of Electrical and Electronic Eng., Imperial College, London, UK, March 1997.

[22] E. C. Levy, "Complex curve fitting," IRE Trans. Autom. Control, pp. 37-43, 1959. 